\title{
Queratitis por Lasiodiplodia theobromae: comunicación de un caso y revisión de la literatura
}

\author{
Margarita Samudio, Florentina Laspina, Norma Fariña, Alicia Franco, \\ Herminia Mino de Kaspar y Gustavo Giusiano
}

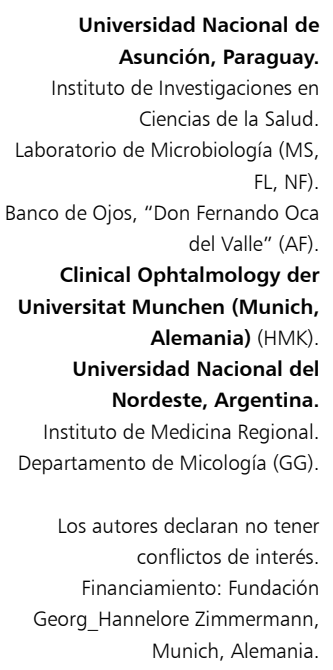

Recibido: 10 de abril de 2014 Aceptado: 30 de octubre de 2014

Correspondencia a: Margarita Samudio microbiologia@iiacs.una.py

\section{Introducción}

L a queratitis infecciosa constituye una de las principales causas de ceguera no reversible en el mundo ${ }^{1}$. Dentro de las etiologías, las de origen fúngico se presentan desde 6 a $60 \%$, dependiendo principalmente de la localización geográfica ${ }^{2-5}$. Se considera una infección oportunista asociada principalmente a trauma ocular con materiales vegetales. Los hongos filamentosos, entre ellos, Fusarium spp. y Aspergillus spp. son los agentes etiológicos de mayor frecuencia ${ }^{2-7}$. En cambio, en países desarrollados la causa principal se relaciona al uso de lentes de contacto, siendo más frecuente la infección por levaduras, como Candida spp $^{6}$.

La queratitis fúngica es una entidad de peor pronóstico que una infección bacteriana y requiere de un diagnóstico precoz para evitar consecuencias irreversibles ${ }^{6,7}$.

\section{Caso clínico}

Paciente agricultor de 60 años de edad, con antecedente de trauma ocular con material vegetal, consultó por una disminución brusca de la agudeza visual de 48 $\mathrm{h}$ de evolución, acompañado de dolor ocular moderado y fotofobia. Al examen oftalmológico, el ojo derecho presentaba una agudeza visual de 20/100 con el segmento anterior normal y el ojo izquierdo con una buena proyección luminosa en los cuatro cuadrantes y una úlcera central de 0,6 x 0,6 mm con bordes difusos. El paciente refirió como co-morbilidad una hipertensión arterial de dos años de evolución.
Se realizó el diagnóstico clínico de una queratitis infecciosa. Se tomó una muestra de la úlcera para estudio microbiológico e inmediatamente se inició tratamiento tópico con fluconazol $0,2 \%$ y moxifloxacina $0,5 \%$ cada 1 hora.

A las $24 \mathrm{~h}$, en el primer control, el examen micológico directo reveló la presencia de elementos fúngicos, por lo que se agregó natamicina $5 \%$ en gotas cada una hora y se espació la administración de moxifloxacina tópica cada 3 h. Como el paciente refirió intenso dolor ocular, se prescribió colirio de atropina, tres veces al día.

En el segundo control, a las 48 h, se observó una hiperemia conjuntival moderada y tres lesiones satélites. En el tercer control, a las $72 \mathrm{~h}$, el paciente refirió dolor ocular muy intenso por lo que se le prescribió nuevamente atropina en gotas. La queratitis y las lesiones satélites siguieron sin cambios, observándose colgajos corneales. Se continuó tratamiento con natamicina y fluconazol tópico y moxifloxacina en gotas, 4 veces al día.

Después de una semana, el paciente refirió menos dolor ocular y se observó ligera mejoría de la lesión, por lo que se suspendió la moxifloxacina. En los seis siguientes controles, las lesiones satélites ya no fueron visibles, hubo disminución de los colgajos corneales, el tamaño de la queratitis disminuyó a 0,5 x $0,5 \mathrm{~mm}$ y el paciente ya no refirió dolor.

Después de 41 días de tratamiento se observó ausencia de actividad en la lesión, sin embargo, persistió la cicatriz corneal.

El último control se realizó tres meses después del traumatismo ocular, momento en que se recomendó 

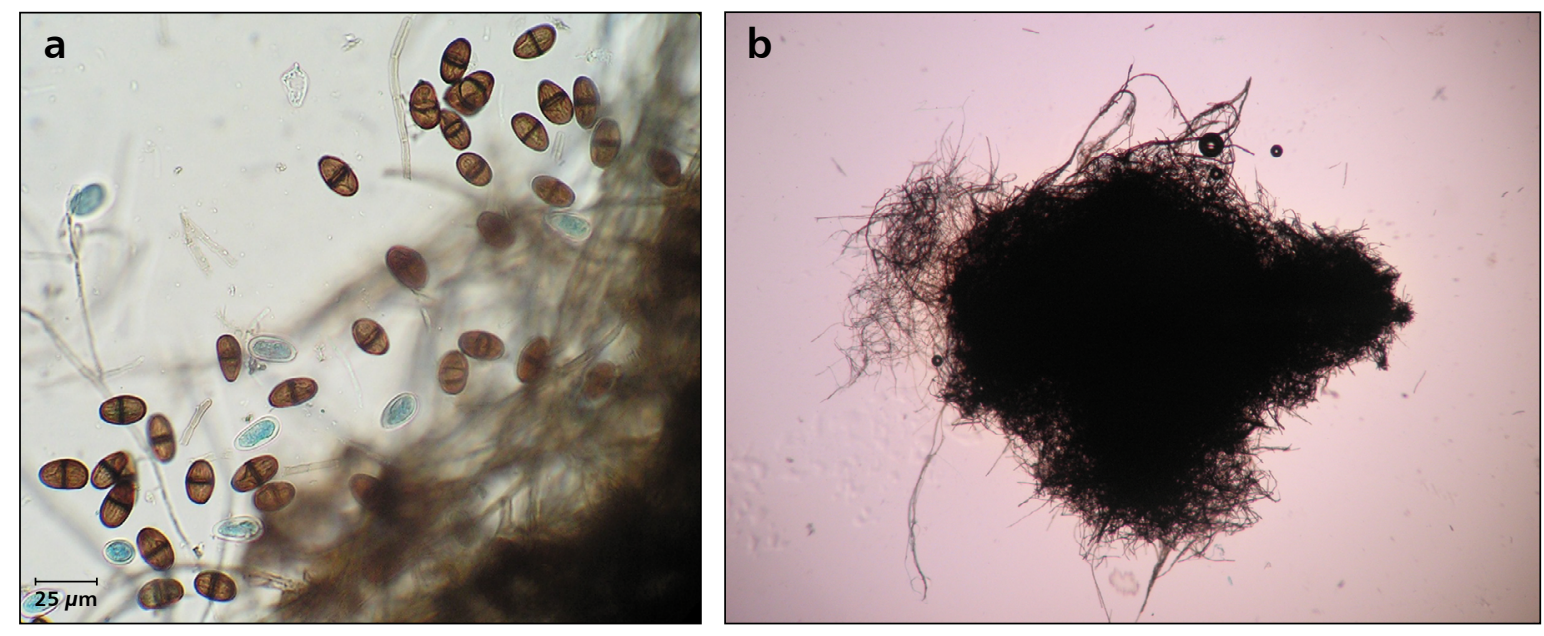

Figura 1. Características de Lasiodiplodia theobromae: a. conidias jóvenes hialinas aceptadas y conidias maduras de $25 \mu \mathrm{m}$, oscuras $\mathrm{y}$ septadas; b. picnidio entre porta y cubreobjetos (10X).

cirugía de cataratas y trasplante de córnea debido a la cicatrización de la córnea.

\section{Estudio microbiológico}

El material de raspado de la lesión corneal fue realizado por el médico oftalmólogo con espátula de Kimura y se envió para estudio microbiológico al Instituto de Investigaciones en Ciencias de la Salud.

En el examen en fresco con $\mathrm{KOH} 10 \%$ (40X) y en la tinción de Gram se observaron hifas septadas ramificadas.

Los cultivos bacterianos se realizaron en caldo tioglicolato a $35^{\circ} \mathrm{C}$ y en agar sangre y agar chocolate en atmósfera de $\mathrm{CO}_{2}$ al $5 \%$ a igual temperatura; todos resultaron negativos.

Los cultivos para hongos se realizaron en agar Sabouraud y agar papa dextrosa y se incubaron a $28^{\circ} \mathrm{C}$. A las $72 \mathrm{~h}$ de incubación se obtuvo desarrollo de colonias de un hongo filamentoso de aspecto flocoso, coloración inicialmente blanca y que se fue oscureciendo con el tiempo tomando finalmente una coloración gris oscura a los 7 días y con abundante micelio aéreo, sin difundir pigmento al medio de cultivo. La colonia fue derivada al Departamento de Micología del Instituto de Medicina Regional de la Universidad Nacional del Nordeste (Argentina) para su identificación. La micro-morfología reveló la presencia de picnidios color café oscuro a negro con paráfisis hialinas septadas. Conidios elipsoidales de $(18-30) \times(10-15) \mu \mathrm{m}$, inicialmente hialinos y no septados que al madurar se observaron de color marrón oscuro con un septo medio y con estrías longitudinales (Figura 1). El aislado fue identificado como Lasiodiplodia theobromae (Pat.) Griffon \& Maubl., según la referencia bibliográfica de Alves y cols ${ }^{8}$.

No se determinó la sensibilidad antifúngica debido a que no se hace de rutina y no hay metodologías de referencia que estandaricen el estudio de sensibilidad para este hongo.

\section{Discusión}

Lasiodiplodia theobromae (Pat.) Griffon \& Maubl. es la especie tipo del género Lasiodiplodia. Es un ascomycete pleomórfico y plurívoro que tiene una amplia distribución geográfica pero con mayor prevalencia en áreas tropicales y subtropicales ${ }^{8}$.

Este agente ha sido reportado como agente causante de sinusitis, neumonía, onicomicosis, absceso glúteo, feohifomicosis subcutánea y de varias patologías oculares, entre ellas, más frecuentemente queratitis y escleritis ${ }^{9-11}$. Sin embargo, L. theobromae sigue siendo una causa excepcional de queratitis micótica con menos de 50 casos publicados en el mundo. En la Tabla 1 se presentan los casos de queratitis humana causada por L. theobromae reportados en la literatura médica ${ }^{11-28}$.

En 1967, Puttanna y cols. ${ }^{12}$ informaron los dos primeros casos de queratitis por este hongo en la India. Posteriormente, surgieron otros reportes también en India; Sri Lanka, sur de los E.U.A y de otros países ${ }^{11,13-28}$ que confirmaron la capacidad patogénica oportunista de este hongo para la córnea humana.

En Brasil, en un estudio retrospectivo de 32 años sobre queratitis en adultos mayores se mencionan dos casos causados por este hongo ${ }^{4}$. En Ghana, L. theobromae ha sido asociada a 5,5\% de las queratitis micóticas y en India entre $0,3 \%$ y $3,9 \%{ }^{21,30}$.

En países tropicales como Paraguay, la incidencia de queratitis por hongos es relativamente alta debido a las condiciones climáticas ${ }^{31}$; sin embargo, el caso que se presenta es la primera comunicación de una queratitis causada por $L$. theobromae. 


\begin{tabular}{|c|c|c|c|c|c|}
\hline Referencia & n de casos & Lugar & Año & Diagnóstico & Resultado del tratamiento \\
\hline Slomovic et al. ${ }^{11}$ & 1 & Florida & 1985 & Panoftalmitis & No reportado \\
\hline Puttanna et al. ${ }^{12}$ & 2 & India & 1967 & Queratitis & No reportado \\
\hline Laverde et al. ${ }^{13}$ & 1 & Colombia & 1973 & Queratitis & No reportado \\
\hline Valenton et al. ${ }^{14}$ & 1 & Filipinas & 1975 & Queratitis & No reportado \\
\hline Rebell et al. ${ }^{15}$ & 4 & Florida & 1976 & Queratitis & 3 buena recuperación y 1 queratoplastía 11 días post natamicina tópica \\
\hline Liesegang et al. ${ }^{16}$ & 5 & Florida & 1980 & Queratitis & No reportado \\
\hline Thomas $^{17}$ & 1 & India & 1991 & Queratitis & queratoplastia \\
\hline Gonawerdena et al. ${ }^{18}$ & 1 & Sri Lanka & 1994 & Queratitis & Tratamiento no fue evaluado \\
\hline Rosa et al. ${ }^{19}$ & 2 & Florida & 1994 & Queratitis & No reportado \\
\hline Dunlop et al. ${ }^{20}$ & 2 & Bangladesh & 1994 & Queratitis & No reportado \\
\hline Hagan et al. ${ }^{21}$ & 6 & Ghana & 1995 & Queratitis & No reportado \\
\hline Borderie et al. ${ }^{22}$ & 1 & Francia & 1997 & Endoftalmitis & Evisceración \\
\hline Srinivasan et al. ${ }^{23}$ & 5 & India & 1997 & Queratitis & No reportado \\
\hline Garg et al. ${ }^{24}$ & 3 & India & 2000 & Queratitis & No reportado \\
\hline Gopinathan et al. ${ }^{25}$ & 7 & India & 2002 & Queratitis & No reportado \\
\hline Donnio et al. ${ }^{26}$ & 1 & Francia & 2006 & Endoftalmitis & Manejo quirúrgico \\
\hline Thew et al. ${ }^{27}$ & 2 & Australia & 2008 & Queratitis & No reportado \\
\hline Saha et al. ${ }^{28}$ & 1 & India & 2012 & Queratitis & Queratoplastía \\
\hline Presente estudio & 1 & Paraguay & 2014 & Queratitis & $\begin{array}{l}\text { Cicatriz corneal con necesidad de trasplante después de tratamiento tópico con natamicina } \\
\text { y fluconazol por } 37 \text { días }\end{array}$ \\
\hline
\end{tabular}

En general las queratitis micóticas pueden ser graves y en ocasiones evolucionan a una endoftalmitis y panoftalmitis. El 82\% de los casos de queratitis causadas por L. theobromae en la India fueron graves y, en los casos no tratados correctamente o en aquellos donde fueron utilizados corticosteroides tópicos, las úlceras producidas por este hongo se diseminaron y comprometieron las capas más profundas de la córnea, con extensión intraocular o la formación de descemetocele ${ }^{32}$. En uno de los casos de queratitis por L. theobromae estudiados en Miami, E.U.A, se observó el hongo en la córnea e invadiendo la membrana de Descemet, requiriendo un tratamiento queratoplástico después del empleo tópico de natamicina por 11 días $^{15}$. Se ha afirmado que $L$. theobromae puede causar una queratitis grave que no responde al tratamiento médico y que requiere queratoplastia penetrante ${ }^{15,22}$. En nuestro caso $L$. theobromae produjo una invasión corneal, con lesiones satélites y colgajos corneales, dejando lesiones cicatriciales con indicación de trasplante de córnea.

En base a la bibliografía revisada, presentada en la Tabla 1, se observa que la infección por este hongo frecuentemente es el resultado de la inoculación directa, sin otro factor agravante asociado. En nuestro caso, el paciente tenía el antecedente de un trauma en la córnea con material vegetal y sólo refería hipertensión arterial como enfermedad subyacente.

La virulencia de los hongos fitopatógenos está relacionada con una serie de mecanismos que les dan la capacidad de adaptarse al hospedero. Estos cambian su morfología, según estímulos físicos y fisiológicos, para lograr la infección y forman estructuras particulares para promover la penetración, como tubos germinativos, apresorios y agregados hifales más complejos. La penetración puede verse favorecida por digestión enzimática ${ }^{33,34}$. Lasiodiplodia theobromae expresa proteínas extracelulares con actividades proteolíticas y moléculas extracelulares con propiedades citotóxicas sobre líneas celulares $\mathrm{Vero}^{34}$. Para el tratamiento de las queratitis se utilizan principalmente tratamientos tópicos, mientras que para las endoftalmitis tratamientos tanto sistémicos como locales ${ }^{35}$. En muchos países el fármaco de elección para las queratitis por hongos filamentosos es la natamicina ${ }^{36}$; sin embargo, se ha reportado una baja tasa de tratamiento exitoso por la escasa penetración ocular del fármaco ${ }^{37}$. Voriconazol, un derivado sintético del fluconazol, se ha convertido en los últimos años en una alternativa prometedora para el tratamiento de esta patología, dada su excelente penetración ocular y su gran espectro antifúngico ${ }^{38}$, siendo el fármaco actualmente recomendado para el tratamiento de queratitis por hongos filamentosos ${ }^{35}$. 
La mayoría de las publicaciones acerca de queratitis producidas por hongos dematiáceos muestran una proporción significativa de casos debidos a $L$. theobromae, sin embargo, no hay información sobre los resultados del tratamiento (Tabla 1). La evaluación de la efectividad a la terapia se basa en reportes de casos. En general, $L$. theobromae tiene escasa respuesta al tratamiento por lo que se requiere finalmente queratoplastia ${ }^{15,17,28}$.

Se ha descrito resistencia in vitro de aislados oculares de L. theobromae a miconazol, ketoconazol, itraconazol, econazol y natamicina, según la revisión realizada por Thomas $^{39}$. En la publicación de Saha y cols. ${ }^{28}$, el aislado de L. theobromae mostró resistencia in vitro a itraconazol y fluconazol y sensibilidad a voriconazol $(1 \mu \mathrm{g} / \mathrm{ml})$ y a anfotericina $\mathrm{B}(4 \mu \mathrm{g} / \mathrm{ml})$; sin embargo, observaron una falta de respuesta clínica al tratamiento inicial con voriconazol tópico, lo que llevó a la progresión de la lesión requiriendo posteriormente una queratoplastia terapéutica. Si bien se ha demostrado que voriconazol tiene buena penetración ocular, es probable que con el tratamiento tópico inicial no se haya alcanzado los valores de CIM a nivel ocular ${ }^{38}$. En nuestro caso, la sensibilidad in vitro no fue realizada y el tratamiento con natamicina y fluconazol controló la infección, sin embargo, el paciente quedó con cicatrices en la córnea con necesidad de trasplante.

En conclusión, L. theobromae es un patógeno oportunista para el ser humano que causa fundamentalmente queratitis micóticas graves las cuales, muchas veces, requieren de procedimientos quirúrgicos y trasplante de córnea. Es muy importante el rápido diagnóstico de estas afecciones con la identificación del agente etiológico a fin de evitar o minimizar consecuencias irreparables.

Agradecimientos. Este trabajo fue realizado dentro de un proyecto apoyado por la Fundación Hannelore-Georg Zimmermann, Munich, Germany.

\section{Resumen}

Se presenta un caso clínico de queratitis causada por Lasiodiplodia theobromae en un agricultor de 60 años de edad, con antecedentes de un trauma ocular con un vegetal. Se realizó un examen oftalmológico con lámpara de hendidura y estudio microbiológico de la úlcera corneal por técnicas microbiológicas convencionales. El examen micológico del raspado corneal reveló la presencia de hifas septadas y el cultivo fue identificado como Lasiodiplodia theobromae. El paciente fue tratado con natamicina al 5\% y fluconazol al 0,2\% durante 37 días. La infección fue controlada, sin embargo, el paciente quedó con cicatrices corneales y con necesidad de trasplante. Es el primer caso de queratitis por Lasiodiplodia theobromae en Paraguay. Se discute la dificultad de manejo de estos casos que a menudo requieren procedimientos quirúrgicos y trasplante de córnea.

\section{Referencias bibliográficas}

1.- Whitcher J P, Srinivasan M, Upadhyay M P. Corneal blindness: a global perspective. Bull World Health Org 2001; 79: 214-21.

2.- Villegas-Flores M, Castellanos-González M, Beltrán Díaz-de la Vega. Análisis de queratitis micóticas en un hospital de tercer nivel. Rev Mex Oftalmol 2012; 86: 231-9.

3.- Cruz Choappa R. Queratitis micótica: identificación de especies en Valparaíso. Bol Micol 2013; 28: 48-52.

4.- Passos R M, Cariello A J, Yu M C, HöflingLima A L. Microbial keratitis in the elderly: a 32-year review. Arq Bras Oftalmol 2010; 73 : 315-9.

5.- $\quad$ Iyer S A, Tuli S S, Wagoner R C. Fungal keratitis: emerging trends and treatment outcomes. Eye Contact Lens 2006; 32: 267-71.

6.- Tuli S S. Fungal keratitis. Clin Ophthalmol 2011; 5: 275-9.

7.- $\quad$ Thomas P A, Leck A K, Myatt M. Characteristic clinical features as an aid to the diagnosis of suppurative keratitis caused by filamentous fungi. Br J Ophthalmol 2005; 89: 1554-8.

8.- Alves A, Crous P W, Correia A, Phillips A J L. Morphological and molecular data reveal cryptic speciation in Lasiodiplodia theobromae. Fungal Diversity 2008; 28: 1-13.

9.- Kindo A J, Pramod C, Anita S, Mohanty S. Maxillary sinusitis caused by Lasiodiplodia theobromae. Indian J Med Microbiol 2010; 28: 167-9.

10.- Woo P C, Lau S K, Ngan A H, Tse H, Tung ET, Yuen K Y. Lasiodiplodia theobromae pneumonia in a liver transplant recipient. J Clin Microbiol 2008; 46: 380-4.

11.- Slomovic A R, Forster R K, Gelender H. Lasiodiplodia theobromae panophthalmitis. Can J Ophthalmol 1985; 20: 225-8.

12.- Puttana S T. Mycotic infections of the cornea. J All India Ophthalmol Soc 1967; 15: 11-8.

13.- Laverde S, Moncada L H, Restrepo A, Vera C L. Mycotic keratitis: 5 cases caused by unusual fungi. Sabouraudia 1973; 11: 119-23.

14.- Valenton M J, Rinaldi M G, and Butler E E. A corneal abscess due to the fungus Botryodiplodia theobromae. Can J Ophthalmol 1975; 10: 416-8.

15.- Rebell G, Forster R K. Lasiodiplodia theobromae as a cause of keratomycoses. Sabouraudia 1976; 14: 155-70.

16.- Liesegang T J, Forster R K. Spectrum of microbial keratitis in South Florida. Am J Ophthalmol 1980; 90: 38-47.
17.- Thomas P A, Garrison R G, Jansen T Intrahyphal hyphae in corneal tissue from a case of keratitis due to Lasiodiplodia theobromae. J Med Vet Mycol 1991; 29: 263-7.

18.- Gonawerdena S A, Ranasinghe K P, Arseculeratne S N, Seimon C R, Ajello L. Survey of mycotic and bacterial keratitis in Sri Lanka. Mycopathologia 1994; 127: 77-81.

19.- Rosa R H Jr, Miller D, Alfonso E C. The changing spectrum of fungal keratitis in South Florida. Ophthalmology 1994; 101: 1005-13.

20.- Dunlop A A, Wright E D, Howlader S A, Nazrul I, Husain R, McClellan K, et al. Suppurative corneal ulceration in Bangladesh: a study of 142 boxes, examining the microbiological diagnosis, clinical and epidemiological features of bacterial and fungal keratitis. Aust N Z J Ophthalmol 1994; 22 : 105-10.

21.- Hagan M, Wright E, Newman M, Dolin P, Johnson G. Causes of suppurative keratitis in Ghana. Br J Ophthalmol 1995; 79: 1024-8.

22.- Borderie V M, Bourcier T M, Poirot J L, Baudrimont M, Prudhomme de Saint-Maur P, Laroche L. Endophthalmitis after Lasiodiplodia theobromae corneal abscess. Graefes Arch Clin Exp Ophthalmol 1997; 235: 259-61.

23.- Srinivasan M, Gonzales C A, George C, 
Cevallos V, Mascarenhas J M, Asokan B, et al. Epidemiology and aetiological diagnosis of corneal ulceration in Madurai, South India. Br J Ophthalmol 1997; 81: 965-71.

24.- Garg P, Gopinathan U, Choudhary K, Rao G N. Keratomycosis: clinical and microbiologic experience with dematiaceous fungi. Ophthalmology 2000; 107: 574-80.

25.- Gopinathan U, Garg P, Fernandes M, Sharma S, Athmanathan S, Rao G N. The epidemiological features and laboratory results of fungal keratitis. A 10-year review at a referral eye care center in South India. Cornea 2002; 21: 555-9.

26.- Donnio A, Desbois N, Boiron P, The'odose R, Mouniee D, Thoumazet F, et al. Mycotic keratitis and endophthalmitis caused by unusual fungi: Lasiodiplodia theobromae. J Fr Ophtalmol 2006; 29: e4.

27.- Thew M R, Todd B. Fungal keratitis in far north Queensland, Australia. Clin Experiment Ophthalmol 2008; 36: 721-4.

28.- Saha S, Sengupta J, Banerjee D, Khetan A. Lasiodiplodia theobromae keratitis: a case report and review of literature. Mycopathologia 2012; 174: 335-9.

29.- Thomas P A, Kalavathy C M, Devanandan P. Lasiodiplodia theobromae keratitis-a clinical profile. J Tamilnadu Ophthalmol Assoc 1998; 39: 31-2.

30.- Leck A K, Thomas P A, Hagan M, Kaliamurthy J, Ackuaku E, John M, et al. Aetiology of suppurative corneal ulcers in Ghana and south India, and epidemiology of fungal keratitis. $\mathrm{Br} \mathrm{J}$ Ophthalmol 2002; 86: 1211-5.

31.- Laspina F, Samudio M, Cibils D, Ta C N, Fariña N, Sanabria R, et al. Epidemiological characteristics of microbiological results on patients with infectious corneal ulcers: a 13-year survey in Paraguay. Graefes Arch Clin Exp Ophthalmol 2004; 242: 204-9.

32.- Stern G A, Buttross M. Use of corticosteroids in combination with antimicrobial drugs in the treatment of infectious corneal disease. Ophthalmology 1991; 98: 847-53.

33.- Struck C. Infection strategies of plant parasitic fungi. En: Cooke B M, Jones G D, Kaye B, eds. The Epidemiology of Plant Diseases, $2^{\text {nd }}$ ed. Dordrecht, the Netherlands: Springer. 2006; 117-37.

34.- Mendgen K, Hahn M, Deising $\mathrm{H}$. Morphogenesis and mechanisms of penetration by plant pathogenic fungi. Annu Rev Phytopathol 1996; 34: 367-86.

35.- Mellado F, Rojas T, Cumsille C. Fungal keratitis: review of diagnosis and treatment. Arq Bras Oftalmol 2013; 76: 52-6.

36.- Natamycin approved-first US drug for fungal keratitis. FDA Drug Bull 1978; 8: 37-8.

37.- O'Day D M, Head W S, Robinson R D, Clanton $\mathrm{J}$ A. Corneal penetration of topical amphotericin B and natamycin. Curr Eye Res 1986; 5: 87782.

38.- Hariprasad S M, Mieler W F, Lin T K, Sponsel W E, Graybill J R. Voriconazole in the treatment of fungal eye infections: A review of current literature. Br J Ophthalmol 2008; 92: 871-8.

39.- Thomas P A. Current perspectives on ophthalmic mycoses. Clin Microbiol Rev 2003; 16: 730-97. 